\title{
COSMIC X-RAY BACKGROUND FROM EARLY ACTIVE GALACTIC NUCLEI
}

\author{
Andrzej A. Zdziarski \\ Space Telescope Science Institute, Baltimore, MD 21218, USA
}

A model for the origin of the cosmic X-ray background (hereafter XRB) is presented. The component of the background left after subtraction of the known classes of sources is explained by emission from a population of black hole sources at the redshift of $z \sim 4-5$. The model is presented in more detail elsewhere (Zdziarski 1988). Here, we summarize its most important results.

The cosmic X-ray background after subtraction of the estimated contributions of Seyfert galaxies and quasars yields a residual spectrum in the $3-50 \mathrm{keV}$ range,

$$
\frac{d F}{d E} \propto E^{-\alpha_{x}} \exp \left(-E / E_{0}\right)
$$

with $23 \mathrm{keV} \leq E_{0} \leq 30 \mathrm{keV}$ and $\left|\alpha_{x}\right| \lesssim 0.2$ (Leiter and Boldt 1982; Setti 1985).

We study here a hypothesis in which the spectrum (1) is due to the contribution from a population of presently unresolved discrete sources (e.g., Leiter and Boldt 1982). The existence of such a population is suggested by Hamilton and Helfand (1987), who found that the quasar spatial density is too small to account for the low amplitude fluctuations in the X-ray counts of the Einstein Deep Survey. The spectrum of an individual source contributing to the residual XRB should follow eq. (1) with $E_{0}=(23-30)(1+z) \mathrm{keV}$ in the rest frame of the source.

In the model, the emission of an individual source is due to a turbulent spherical accretion flow (Mészáros 1975). The gravitational energy is used to heat thermal electrons in the flow and to generate magnetic fields in equipartition with the flow energy density. The emitted spectrum, which is self-consistently solved for, is due to Compton upscattering by the thermal electrons of cyclo-synchrotron photons emitted by the same electrons and partially self-absorbed. The $\mathrm{X}$-ray spectrum is found to have an approximately constant spectral index, $\alpha_{x} \simeq 0$, in the wide range of the dimensionless mass accretion rate of $0.5 \gtrsim \dot{m} \gtrsim 20$. Here $\dot{m} \equiv \dot{M} c^{2} / L_{\mathrm{E}}, \dot{M}$ is the mass accretion rate, and $L_{\mathrm{E}}$ is the Eddington luminosity. The spectra are cut off above $\sim 2 k T \sim$ const $\sim 150 \mathrm{keV}$ in the range of $\dot{m}$ specified above. Such spectra closely resemble that of the residual cosmic XRB if $z \sim 4$.

This stability of the spectral parameters with the changing accretion rate is critical for our model. The approximate constancy of $k T \sim 10^{2} \mathrm{keV}$ is explained in part by the thermostatic effect of thermal $e^{+} e^{-}$pair production (e.g., Leiter and Boldt 1982; Svensson 1984). The spectral index of $\sim 0$ is characteristic of saturated Comptonization. The Comptonization process is saturated because of the relative deficiency of the self-absorbed thermal cyclo-synchrotron photons.

The model requires that the sources no longer radiate the $\alpha_{x} \sim 0$ spectrum at $z \lesssim 4$. This change of the character of the sources can be associated, for example, with the accretion 
rate becoming sub-Eddington ( $\dot{m} \lesssim 1$ ), which results in an onset of nonthermal processes (Cavaliere and Morrison 1980), which then steepen the X-ray spectra. In the process of spherical accretion, the physical mass accretion rate is not Eddington-limited, as the superEddington part of the photon flux is advected to the black hole (Begelman 1979). If the rate of matter supply, $\dot{M}$, was constant for some initial period, we find that $\dot{m}=t_{\mathrm{E}} / t$, where $t_{\mathrm{E}} \simeq 5 \times 10^{8}$ yrs is the Eddington time. Thus, $\dot{m}$ becomes $\leq 1$ after one Eddington time and the onset of nonthermal processes is expected then. If the sources were formed at $z \gg 1, t(z=4) \simeq t_{\mathrm{E}}$ at $\Omega=1$ and $H_{0}=100 \mathrm{~km} \mathrm{~s}^{-1} \mathrm{Mpc}^{-1}$. Thus, those cosmological parameters are most consistent with our model.

In a detailed cosmological evolution model of the turbulent accreting sources formed at $z_{0}=6$, we found $\alpha_{x}=0.2 \pm 0.1$ for $2.6 \lesssim z \leqslant 5.6$. The observed spectral break energy, $E_{0}$, was found to be between 20 and $40 \mathrm{keV}$ for $3.9 \lessgtr z \leqslant 5.6$ (see Fig. 4 in Zdziarski 1988). Thus, emission coming from a relatively large range of the redshift could give rise to the residual $X R B$ spectrum of eq. (1).

If the sky angular density of the sources contributing to the XRB is $\geq 5000 \mathrm{deg}^{-2}$ (Hamilton and Helfand 1987), the comoving source density is $\geq$ the estimated density of Seyfert I\&II galaxies (Persic et al. 1988). If $H_{0}=100 \mathrm{~km} \mathrm{~s}^{-1} \mathrm{Mpc}^{-1}$, the accumulated mass at $z=4$ is $\leq 5 \times 10^{7} M_{\odot}$, and $L_{x} \leq 5 \times 10^{43} \mathrm{erg}$ sec. Such low-luminosity sources would be at the sensitivity limit of AXAF.

\section{REFERENCES}

Begelman, M. C. 1979, M. N. R. A. S., 187, 237.

Cavaliere, A., and Morrison, P. 1980, Ap. J., 238, L63.

Hamilton, T. T., and Helfand, D. J. 1987, Ap. J., 318, 93.

Leiter, D., and Boldt, E. 1982, Ap. J., 260, 1.

Mészáros, P. 1975, Nature, $258,583$.

Persic, M. et al. 1988, Ap. J., in press.

Setti, G. 1985, in Non-Thermal and Very High Temperature Phenomena in X-Ray Astronomy, ed. G. C. Perola and M. Salvati, (Rome), p. 159.

Svensson, R. 1984, M. N. R. A. S., 209, 175.

Zdziarski, A. A. 1988, M. N. R. A. S., 233, 739.

\section{DISCUSSION}

Dan Schwartz: I challenge the residual spectrum that you fit in your model. I believe it is an artifact of subtracting an exact power law over many decades.

Andrzej Zdziarski: The exact form of the residual spectrum is inded not known at present (cf. the uncertainties in the parameters $\alpha_{x}$ and $E_{0}$ of eq. [1]). The model of this work predicts spectra with $\alpha_{x}$ and $E_{0}$ adjustable to certain extent by its free parameters.

Jerzy Madej: Could you explain in more detail the way you computed X-ray spectra of black hole sources?

Andrzej Zdziarski: A one zone approximation has been used. The part of the source where photons are not advected into the central black hole is assumed to be a homogeneous cloud, in which the supplied power equals the total luminosity. Comptonization is treated using a diffusion approximation. See Zdziarski (1988) for more detail. 